\title{
The burden of carotid-related strokes
}

Kosmas I. Paraskevas ${ }^{1}$, Dimitri P. Mikhailidis ${ }^{2}$, Hediyeh Baradaran ${ }^{3}$, Reinoud P. H. Bokkers ${ }^{4}$, Alun H. Davies ${ }^{5}$, Hans-Henning Eckstein ${ }^{6}$, Gianluca Faggioli ${ }^{7}$, Jose Fernandes E Fernandes ${ }^{8}$, Mauro Gargiulo $^{7}$, Arkadiusz Jawien ${ }^{9}$, Mateja K. Jezovnik ${ }^{10}$, Stavros K. Kakkos ${ }^{11}$, Michael Knoflach ${ }^{12}$, M. Eline Kooi ${ }^{13,14}$, Gaetano Lanza ${ }^{15}$, Christos D. Liapis ${ }^{16}$, Ian M. Loftus ${ }^{17}$, Armando Mansilha ${ }^{18}$, Laura Mechtouff ${ }^{19}$, Antoine Millon ${ }^{20}$, Piotr Myrcha ${ }^{21}$, Andrew N. Nicolaides ${ }^{22}$, Rodolfo Pini ${ }^{7}$, Pavel Poredos ${ }^{23}$, Jean-Baptiste Ricco ${ }^{24}$, Tatjana Rundek ${ }^{25}$, Luca Saba ${ }^{26}$, Mauro Silvestrini ${ }^{27}$, Francesco Spinelli ${ }^{28}$, Francesco Stilo ${ }^{28}$, Sherif Sultan ${ }^{29}$, Jasjit S. Suri ${ }^{30}$, Alexei V. Svetlikov ${ }^{31}$, Tissa Wijeratne ${ }^{32}$, Clark J. Zeebregts ${ }^{33}$, Peter Gloviczki ${ }^{34}$

${ }^{1}$ Department of Vascular Surgery, Central Clinic of Athens, Athens, Greece; ${ }^{2}$ Department of Clinical Biochemistry, Royal Free Hospital Campus, University College London Medical School, University College London (UCL), London, UK; ${ }^{3}$ Department of Radiology and Imaging Sciences, Neuroradiology Division, University of Utah Health, Salt Lake City, UT, USA; ${ }^{4}$ Department of Radiology, Medical Imaging Center, University Medical Center Groningen, Groningen, The Netherlands; ${ }^{5}$ Section of Vascular Surgery, Imperial College \& Imperial Healthcare NHS Trust, London, UK; ${ }^{6}$ Department for Vascular and Endovascular Surgery, Klinikum rechts der Isar, Technical University of Munich, Munich, Germany; ${ }^{7}$ Vascular Surgery, Department of Experimental, Diagnostic and Specialty Medicine, University of Bologna, IRCSS Policlinico di Sant'Orsola, Bologna, Italy; ${ }^{8}$ Department of Vascular Surgery, University of Lisbon, Lisbon Academic Medical Center, Lisbon, Portugal; ${ }^{9}$ Department for Vascular Surgery and Angiology, L. Rydygier Collegium Medicum in Bydgoszcz, Nicolaus Copernicus University in Torun, Poland; ${ }^{10}$ Department of Advanced Cardiopulmonary Therapies and Transplantation, The University of Texas Health Science Centre at Houston, Houston, TX, USA; ${ }^{11}$ Department of Vascular Surgery, University of Patras Medical School, Patras, Greece; ${ }^{12}$ Department of Neurology, Medical University of Innsbruck, Innsbruck, Austria; ${ }^{13}$ CARIM School for Cardiovascular Diseases, Maastricht University, Maastricht, The Netherlands; ${ }^{14}$ Department of Radiology and Nuclear Medicine, Maastricht University Medical Center, Maastricht, The Netherlands; ${ }^{15}$ Vascular Surgery Department, IRCSS MultiMedica Hospital, Castellanza, Italy; ${ }^{16}$ Athens Vascular Research Center, Athens, Greece; ${ }^{17}$ St. George's Vascular Institute, St. George's University London, London, UK; ${ }^{18}$ Faculty of Medicine of the University of Porto, Porto, Portugal; ${ }^{19}$ Stroke Center, Hospices Civils de Lyon, Lyon, France; ${ }^{20}$ Vascular Surgery Department, Hospices Civils de Lyon, Lyon, France; ${ }^{21}$ First Chair and Department of General and Vascular Surgery, Faculty of Medicine, Medical University of Warsaw, Warsaw, Poland; ${ }^{22}$ Department of Surgery, University of Nicosia Medical School, Nicosia, Cyprus; ${ }^{23}$ Department of Vascular Disease, University Medical Centre Ljubljana, Slovenia; ${ }^{24}$ Department of Clinical Research, University of Poitiers, CHU de Poitiers, Poitiers, France; ${ }^{25}$ Department of Neurology, Miller School of Medicine, University of Miami, Miami, FL, USA; ${ }^{26}$ Department of Radiology, Azienda Ospedaliera Universitaria Di Cagliari, Cagliari, Italy; ${ }^{27}$ Neurological Clinic, Department of Experimental and Clinical Medicine, Marche Polytechnic University, Ancona, Italy; ${ }^{28}$ Vascular Surgery Division, Campus Bio-Medico University of Rome, Rome, Italy; ${ }^{29}$ Western Vascular Institute, Department of Vascular and Endovascular Surgery, University Hospital Galway, National University of Ireland, Galway, Ireland; ${ }^{30}$ Stroke Diagnosis and Monitoring Division, AtheroPointTM, Roseville, USA; ${ }^{31}$ Division of Vascular and Endovascular Surgery, North-Western Scientific Clinical Center of Federal Medical Biological Agency of Russia, St. Petersburgh, Russia; ${ }^{32}$ Department of Neurology, Sunshine Hospital and Melbourne Medical School, St. Albans, Victoria, Australia; ${ }^{33}$ Division of Vascular Surgery, Department of Surgery, University Medical Center Groningen, University of Groningen, Groningen, The Netherlands; ${ }^{34}$ Division of Vascular and Endovascular Surgery, Mayo Clinic, Rochester, MN, USA

Correspondence to: Kosmas I. Paraskevas, MD. Department of Vascular Surgery, Central Clinic of Athens, 24, Alex. Papagou street, N. Iraklio 14122, Athens, Greece. Email: paraskevask@hotmail.com.

Response to: Beach KW. Preventing carotid stroke. Ann Transl Med 2021. doi: 10.21037/atm-21-6296

Submitted Nov 23, 2021. Accepted for publication Dec 24, 2021.

doi: 10.21037/atm-2021-12

View this article at: https://dx.doi.org/10.21037/atm-2021-12 
We would like to thank Dr. Beach for his interest in our work published in two previous issues of this Journal on prevention and treatment of stroke due to carotid artery stenosis $(1,2)$. We agree with him that identifying high-risk patients with asymptomatic carotid stenosis (AsxCS) and establishing measures to prevent the catastrophic sequelae of carotid-related strokes should become our research priority.

Stroke risk in patients with AsxCS varies considerably. The Asymptomatic Carotid Stenosis and Risk of Stroke (ACSRS) study assessed the risk of stroke in patients with mild (50-69\%; $\mathrm{n}=198)$, moderate $(70-89 \% ; \mathrm{n}=598)$ and severe AsxCS (90-99\%; $\mathrm{n}=325)$ followed for a mean period of 48 months (range, 6-96) (3). The cumulative 5 -year risks of ipsilateral cerebral or retinal ischemic (CORI) events were $9 \%, 15 \%$ and $20 \%$, respectively (log-rank $\mathrm{P}=0.009)(3)$. The stroke risk varied by different criteria applied, e.g., using carotid plaque area, the presence of juxtaluminal black area without a visible echogenic cup, the presence of silent embolic infarcts on brain computed tomography (CT) scans, etc. For example, for patients with 70-89\% AsxCS, the incidence of stroke was $5.7 \%$ in individuals with plaque types $1-3$ and $0.8 \%$ in those with types 4 and 5 (3). Furthermore, for individuals with 90-99\% AsxCS, the incidence of stroke was $7.7 \%$ in patients with plaque types $1-3$ vs. $0 \%$ in those with types 4 and 5 (3). Based on a stroke risk calculation model, the predicted annual average stroke rate varied from $<1.0 \%$ to $>6.0 \%$ (3).

Dr Beach supports the implementation of selective screening programs in primary care to identify patients with AsxCS. Support for such screening programs targeting high-risk patient subgroups has also been provided from earlier independent reports (4,5). The 2017 European Society for Vascular Surgery (ESVS) guidelines for the management of patients with carotid and vertebral artery stenosis also addressed the issue of screening for AsxCS (6). About $10-15 \%$ of all first-ever strokes occur following thromboembolism from a previously untreated significant AsxCS. Successful stroke prevention strategies could therefore have considerable clinical, social and financial benefits (6). Although both the 2017 ESVS (6) and the 2021 Society for Vascular Surgery (SVS) Guidelines (7) recommended against routine population screening for AsxCS, they recommended that selective screening for AsxCS may be considered in patients with multiple vascular risk factors (e.g., smoking, hypertension, hypercholesterolemia) (Class IIb, Level of Evidence: C). The rationale supporting screening in such high-risk patients is not necessarily to offer a prophylactic carotid intervention, but rather to identify those individuals with AsxCS in order to optimize risk factor control and to provide best medical therapy (BMT). These measures should reduce cardiovascular morbidity/mortality and prevent both strokes and myocardial infarctions/cardiovascular events $(6,7)$.

A crucial issue in the management of AsxCS patients -which is sometimes under-recognized-is patient preference and individual patient needs (8). Patients may have a different perception of their disease and different expectations than their treating physicians (8). Individual patient needs/patient preferences, compliance with BMT, patient age, gender and individual comorbidities are important parameters that should be taken into account when advising patients with AsxCS about the possible treatment options (8). Such patient characteristics support the view that the management of AsxCS should be individualized and tailored to each patient's needs/ expectations (8).

Despite the release of $>30$ national and international guidelines, the optimal management of patients with AsxCS still remains the subject of extensive debate (9). Several plaque and brain imaging biomarkers have been suggested for stroke risk stratification in patients with AsxCS, including change in AsxCS degree of stenosis, plaque volume, plaque echolucency, plaque area, intraplaque hemorrhage, a lipidrich necrotic core, a thin fibrous plaque, microembolic signals in transcranial Doppler, neovascularization, cerebrovascular reserve, silent brain infarcts, and others $(6,10,11)$. The use of these imaging/clinical criteria is essential for stroke risk stratification and for targeting prophylactic carotid revascularization procedures (carotid endarterectomy/carotid artery stenting) to those AsxCS patients that will most likely benefit from them.

\section{Acknowledgments}

Funding: None.

\section{Footnote}

Provenance and Peer Review: This article was commissioned by the editorial office, Annals of Translational Medicine. The article did not undergo external peer review.

Conflicts of Interest: All authors have completed the ICMJE uniform disclosure form (available at https://atm. amegroups.com/article/view/10.21037/atm-2021-12/coif). DPM receives royalties and fees from SAGE, Informa and 
Bentham Science publishers, consulting fees from Novo Nordisk, lecture fees and travel expenses from Amgen and Novo Nordisk. The other authors have no conflicts of interest to declare.

Ethical Statement: The authors are accountable for all aspects of the work in ensuring that questions related to the accuracy or integrity of any part of the work are appropriately investigated and resolved.

Open Access Statement: This is an Open Access article distributed in accordance with the Creative Commons Attribution-NonCommercial-NoDerivs 4.0 International License (CC BY-NC-ND 4.0), which permits the noncommercial replication and distribution of the article with the strict proviso that no changes or edits are made and the original work is properly cited (including links to both the formal publication through the relevant DOI and the license). See: https://creativecommons.org/licenses/by-nc-nd/4.0/.

\section{References}

1. Carotid Artery Stenosis and Stroke: Prevention and Treatment Part I. 2020. Ann Transl Med. Available online: https://atm.amegroups.com/post/view/carotid-arterystenosis-and-stroke-prevention-and-treatment-part-i

2. Carotid Artery Stenosis and Stroke-Prevention and Treatment Part II. 2021. Ann Transl Med. Available online: https://atm.amegroups.com/post/view/carotidartery-stenosis-and-stroke-prevention-and-treatmentpart-ii

3. Paraskevas KI, Nicolaides AN, Kakkos SK. Asymptomatic Carotid Stenosis and Risk of Stroke (ACSRS) study: what

Cite this article as: Paraskevas KI, Mikhailidis DP, Baradaran H, Bokkers RPH, Davies AH, Eckstein HH, Faggioli G, Fernandes E Fernandes J, Gargiulo M, Jawien A, Jezovnik MK, Kakkos SK, Knoflach M, Kooi ME, Lanza G, Liapis CD, Loftus IM, Mansilha A, Mechtouff L, Millon A, Myrcha P, Nicolaides AN, Pini R, Poredos P, Ricco JB, Rundek T, Saba L, Silvestrini M, Spinelli F, Stilo F, Sultan S, Suri JS, Svetlikov AV, Wijeratne T, Zeebregts CJ, Gloviczki P. The burden of carotidrelated strokes. Ann Transl Med 2022;10(3):159. doi: 10.21037/ atm-2021-12 have we learned from it? Ann Transl Med 2020;8:1271.

4. Lavenson GS Jr, Andersen CA. The quick carotid scan for prevention of strokes due to carotid artery disease. Ann Transl Med 2021;9:1202.

5. Paraskevas KI, Eckstein HH, Mikhailidis DP, et al. Rationale for screening selected patients for asymptomatic carotid artery stenosis. Curr Med Res Opin 2020;36:361-5.

6. Naylor AR, Ricco JB, de Borst GJ, et al. Editor's Choice - Management of Atherosclerotic Carotid and Vertebral Artery Disease: 2017 Clinical Practice Guidelines of the European Society for Vascular Surgery (ESVS). Eur J Vasc Endovasc Surg 2018;55:3-81.

7. AbuRahma AF, Avgerinos ED, Chang RW, et al. Society for Vascular Surgery clinical practice guidelines for management of extracranial cerebrovascular disease. J Vasc Surg 2022;75:4S-22S.

8. Paraskevas KI, Mikhailidis DP, Baradaran H, et al. Management of Patients with Asymptomatic Carotid Stenosis May Need to Be Individualized: A Multidisciplinary Call for Action. J Stroke 2021;23:202-12.

9. Paraskevas KI, Mikhailidis DP, Antignani PL, et al. Optimal Management of Asymptomatic Carotid Stenosis in 2021: The Jury is Still Out. An International, Multispecialty, Expert Review and Position Statement. J Stroke Cerebrovasc Dis 2022;31:106182.

10. Donners SJA, Toorop RJ, de Kleijn DPV, et al. A narrative review of plaque and brain imaging biomarkers for stroke risk stratification in patients with atherosclerotic carotid artery disease. Ann Transl Med 2021;9:1260.

11. Kassem M, Florea A, Mottaghy FM, et al. Magnetic resonance imaging of carotid plaques: current status and clinical perspectives. Ann Transl Med 2020;8:1266. 